\title{
A comparative study on the hydrodynamics of liquid-liquid hydrocyclonic separation
}

\author{
H. H. Al-Kayiem, H. Osei, K. Y. Yin \& F. M. Hashim \\ Mechanical Engineering Department, \\ Universiti Teknologi PETRONAS, Malaysia
}

\begin{abstract}
The productivity of wells with a higher water-oil ratio can be improved by the use of a liquid-liquid hydrocyclone separator in the downhole. This system presents an excellent water flooding technique as the water separated downhole is pumped back into the same formation. The produced fluid undergoes a lot of orientations and motions from the time it enters the hydrocyclone until it exits the outlets. This paper helps bring to light the hydrodynamics of the liquid-liquid hydrocyclones, explaining how the fluid behaves once it is within the hydrocyclone, in order to achieve efficient separation. Analytical solutions were used to analyse some of the important flow pattern phenomenon within the liquid-liquid hydrocyclone. The fluid properties considered in this work are similar to that of the Malaysian Bayan offshore field. The results showed that the flow reversal radius was paramount in determining the separation efficiency. There were two existing swirling zones. In the inner zone the light fluid fraction is spiralling upwards, while in the outer zone the heavier fluid fraction is spiralling downward against the walls of the hydrocyclone.
\end{abstract}

Keywords: hydrocyclone, oil/water separation, downhole separation, liquid-liquid separator.

\section{Introduction}

The hydrocyclone system has been used over the years in industrial separation due to its simple design, easy operation, low cost and low maintenance (Chen et al. [1]). It thus finds many applications in the separation of solid-liquid streams, liquid-liquid streams and gas-liquid streams (Bowers et al. [2]). Liquid-liquid hydrocyclones (LLHCs) are those employed in the separation of 
liquid-liquid streams. Due to advanced technology, LLHC can now be installed downhole (inside the well) to separate the oil-water product, due to its compactness, absence of moving parts and no chemical additives. This technology is a cost effective method to handle the increased water production in oil wells by separating the produced water downhole and re-injecting it into the same formation at suitable depths. Thus it provides the platform for reduction in the volume of water produced to the surface, reduction in water treatment cost and prevention of pollution at the surface. The fluid that enters the hydrocyclone has to undergo some flow patterns in order to bring about a successful separation.

The purpose of this study is to understand how the fluids flow and behave within the cyclone. It also presents some important factors that help influence the separation process within LLHC. For that, the flow behaviour within the cyclone boundaries was simulated using the mathematical models available in the literature that describe the hydrodynamics of the LLHC. The fluid properties and the production candidates were adopted from one of the Malaysian offshore fields. The results are presented in terms of the three flow velocity components; radial, axial, and tangential within the hydrocyclone.

\section{The LLHC basis}

The diagram in fig. 1(a) illustrates a typical hydrocyclone, while fig. 1(b) is representing the modified LLHC according to Gomez et al. [3] known as double cone hydrocyclone. The analysis of this paper is adopting the double cone cyclone since it has been proven to be more efficient for liquid-liquid separation. The geometries of the hydrocyclone adopted for the present simulation are outlined in fig. 1(b) and given in table 1. The mixed fluid under pressure is administered through the tangential inlets of the hydrocyclone. As the fluid is forced down the liner, the first tapering design (top cone) accelerates the fluids in the narrowing cross sectional area, thus developing the high centrifugal forces required to separate the fluids. This rapid acceleration is then maintained in the fine tapered section (down cone), which is designed to offset centrifugal and frictional losses (Schubert [4]). The spinning of the fluid develops a vortex within the body of the cyclone thereby creating a strong gravity field. Fluids with different densities in this gravity field move in radial opposite directions (Husveg et al. [5]). The swirling movement of the fluid increases the centrifugal force which causes the heavier water fraction to migrate to the walls of the cyclone whereas the lighter oil fraction is displaced to form a low central core $[2,3]$. Thus the fluids are segregated based on density difference. Gomez et al. [3] pointed out that there is the existence of both forced and free vortexes - the forced vortex in the region close to the axis of the LLHC with the free-like vortex existing at the outer region. These vortexes influence the separation within the LLHC. The outer vortex moves downwards carrying part of the fluid to the underflow outlet while the inner vortex flows in a reverse direction carrying the other part of the light fluid to the overflow outlet. The reverse flow in the LLHC is due to the high swirling intensity at the inlet which increases the 
centrifugal force and causes the pressure to be high near the wall region and very low toward the core region. By maintaining the underflow outlet at a higher pressure than that of the overflow, the concentrated light fluid core of the vortex is forced to flow counter-current to the main flow $[2,3]$. The centrifugal force developed accelerates the settling rate of the fluid particles thereby separating them according to size, shape, and difference in density. Due to the action of the drag force, the oil moves to the core region of the LLHC and is caught by the reverse flow to be separated through the vortex finder. The water moves to the walls of the LLHC and migrates to the apex opening $[3,6]$.

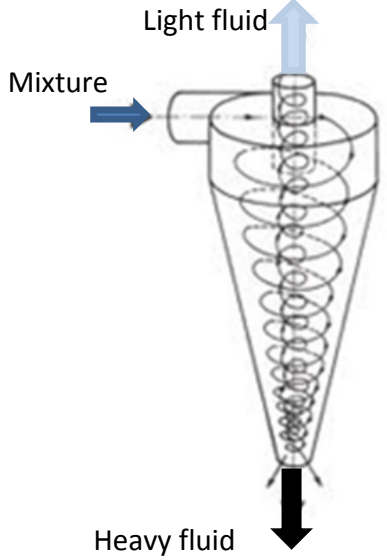

(a) Typical hydrocyclone [Courtesy of Wikipedia].

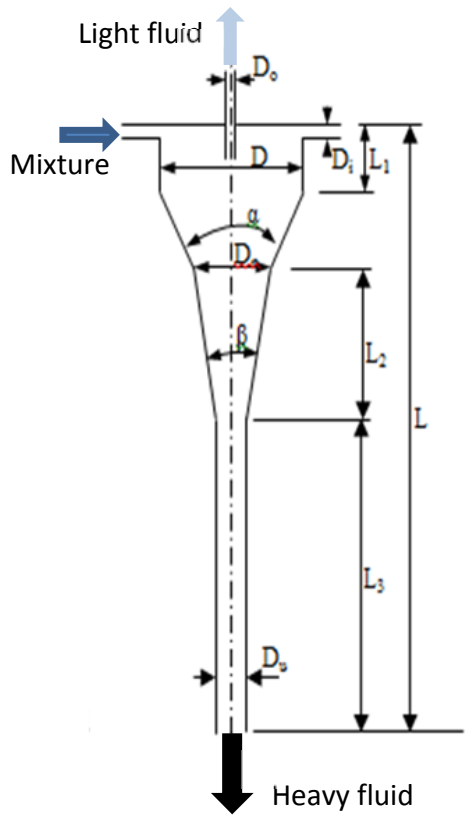

(b) Double cone hydrocyclone [3].

Figure 1: Outlines of the hydrocyclone separator. (a) Typical configuration, (b) Modified double cone configuration.

Table 1: The geometries of the simulated hydrocyclone as suggested by [3].

\begin{tabular}{|c|c|c|c|c|}
\hline \multirow{2}{*}{ Diameters } & $D_{i} / D$ & $D_{u} / D$ & $D_{c} / D$ & $D_{o} / D$ \\
\cline { 2 - 5 } & 0.175 & 0.25 & 0.5 & 0.05 \\
\hline \multirow{2}{*}{ Lengths } & $L_{1} / D$ & $L_{2} / D$ & $L / D$ & $L_{3} / D$ \\
\cline { 2 - 5 } & 1 & 3 & 20 & 15 \\
\hline \multirow{2}{*}{ Angles } & $\alpha$ & $\beta$ & & \\
\cline { 2 - 5 } & $20^{\circ}$ & $1.5^{\circ}$ & & \\
\hline
\end{tabular}




\section{Swirl intensity}

The swirl intensity, $\Omega$, influences the centrifugal force needed for the separation of the fluid within the LLHC and it is produced by the tangential inlet(s) of the LLHC. It is defined as 'the ratio of the local tangential momentum flux to the total momentum flux' (Caldentey et al. [6]). However, in order to better understand the behaviour of the swirl intensity so as to better predict it, some studies have been carried out which resulted in some correlations that can be used to know the profile of the swirl intensity within the LLHC. Chang and Dhir [7] came up with the correlation shown in Eq. 1 which took into consideration only the momentum flux at both the inlet slot and the characteristic diameter position.

$$
\Omega=1.48\left(\frac{M_{t}}{M_{T}}\right)^{0.93} \operatorname{EXP}\left[-0.113\left(\frac{M_{t}}{M_{T}}\right)^{0.35}\left(\frac{z}{D_{c}}\right)^{0.7}\right] \quad \text { for } \frac{z}{D_{c}} \geq 2
$$

Mantilla [8] has incorporated the fluid properties and the inlet configuration and ended up with Eq. 2.

$$
\Omega=1.48\left(\frac{M_{t}}{M_{T}} I^{2}\right)^{0.93} \operatorname{EXP}\left[-\left(\frac{1}{2}\right)\left(\frac{M_{t}}{M_{T}} I^{4}\right)^{0.35}\left(\frac{1}{\mathrm{Re}}\right)^{0.16}\left(\frac{z}{D c}\right)^{0.7}\right] \quad \text { for } \frac{z}{D_{c}} \geq 2
$$

Caldentey et al. [6] correlation shown in Eq. 3 included the semi angle, $\beta$, of the LLHC.

$$
\begin{gathered}
\Omega=1.48\left(\frac{M_{t}}{M_{T}} I^{2}\right)^{0.93}\left(1+1.2 \tan (\beta)^{0.15}\right) * \\
E X P\left[-\left(\frac{1}{2}\right)\left(\frac{M_{t}}{M_{T}} I^{4}\right)^{0.35}\left(\frac{1}{\operatorname{Re}_{z}}\right)^{0.16}\left(\frac{z}{D c}\right)^{0.7}\left(1+1.2 \tan (\beta)^{0.12}\right)\right]
\end{gathered}
$$

By combining Caldentey and Erdal correlations, Gomez [9] ended up with Eq. 4 as the correlation for swirl intensity

$$
\begin{aligned}
& \Omega=0.49 \operatorname{Re}^{0.118}\left(\frac{M_{t}}{M_{T}} I^{2}\right)^{0.93}\left(1+1.2 \tan (\beta)^{0.15}\right) * \\
& E X P\left[-\left(\frac{1}{2}\right)\left(\frac{M_{t}}{M_{T}} I^{4}\right)^{0.35}\left(\frac{1}{\operatorname{Re}_{z}}\right)^{0.16}\left(\frac{z}{D c}\right)^{0.7}\left(1+2 \tan (\beta)^{0.12}\right)\right]
\end{aligned}
$$

where 


$$
\begin{array}{r}
\operatorname{Re}_{z}=\frac{\rho_{c} U_{a v z} D_{z}}{\mu_{c}} \\
\frac{M_{t}}{M_{T}}=\frac{A_{c}}{A_{i s}}
\end{array}
$$

For Eq. 2 and Eq. 4, $I=1-\exp \left(-\frac{n}{2}\right)$, and for Eq. 3, $I=1-\exp (-n)$.

$\rho_{c}$ and $\mu_{c}$ are the density and viscosity of the continuous phase respectively; $z$ is the axial position; $A_{c}$ and $A_{i s}$ in Eq. 6 are the cross-sectional area at the characteristic diameter $D_{c}$ and at the inlet slot respectively; $U_{a v z}$ is the average axial velocity at $z$. For Eq. 3 and Eq. 4, $\mathrm{n}=1.5$ for twin inlets and for Eq. 2, $\mathrm{n}=2$ for twin inlets.

\section{Velocity field predictions}

The velocity profiles can be predicted once the swirl intensity can be known and predicted for specific axial positions. This is because the swirl intensity by definition is relating the local axial and tangential velocities $[6,10]$.

\subsection{Axial velocity}

The axial velocity profile has been known to follow a third-order polynomial equation with proper boundary conditions. It can therefore be predicted using Eq. (7) as shown below [6]:

$$
\begin{gathered}
\frac{u(r)}{U_{a v z}}=\frac{2}{C}\left(\frac{r}{R_{z}}\right)^{3}-\frac{3}{C}\left(\frac{r}{R_{z}}\right)^{2}+\frac{0.7}{C}+1 \\
C=\left(\frac{r_{r e v}}{R_{z}}\right)^{2}\left(3-2\left(\frac{r_{r e v}}{R_{z}}\right)\right)-0.7 \\
\frac{r_{r e v}}{R_{z}}=0.21 \Omega^{0.3}
\end{gathered}
$$

The boundary conditions considered are maximum velocity at the wall; zero velocity at the location of reverse flow; velocity been symmetric about the LLHC axis and assumption of mass conservation.

\subsection{Tangential velocity}

It has been confirmed experimentally that, the tangential velocity is a combination of a forced vortex near the hydrocyclone axis, and a free vortex in the outer wall region, neglecting the effect of the wall boundary layer. The tangential velocity influences the swirling movement of the fluid thereby 
influencing the centrifugal forces needed to cause segregation of the fluid based on density difference. The tangential velocity profile can be predicted by the use of the following equation $[6,10]$ :

$$
\frac{w}{U_{a v c}}=\frac{T_{m}}{\left(r / R_{c}\right)}\left\{1-\exp \left[-B\left(\frac{r}{R_{c}}\right)^{2}\right]\right\}
$$

where, $U_{a v c}$ and $R_{c}$ are the average axial velocity and the radius at the characteristic diameter, $D_{c}$, respectively; and $\mathrm{r}$ is the radial position.

$$
T_{m}=\Omega ; \quad B=245.8 \Omega-2.35 \text { (Twin inlets) }
$$

\subsection{Radial velocity}

The radial velocity, $\mathrm{v}$, of the continuous phase can also be predicted by the use of Eq. (11). However, the radial velocity is frequently much lesser in magnitude than the other velocity components and has been assumed negligible in many previous works (e.g. Caldentey et al. [6] and Amini et al. [10]).

$$
v=-\frac{r}{R_{z}} u \tan (\beta)
$$

\section{Results and discussion}

The predictions are carried out taking the cylinder diameter $D_{c}=30 \mathrm{~mm}$. the fluid properties are laboratory measurement results of crude from offshore field. The prediction results using five different models are presented graphically with inclusion of one set experimental data from Amini et al. [10] and called here as data set 4. Another set of experimental data, belongs to Colman and Thew, extracted from Gomez [9], is also considered.

Fig. 2 shows how the swirl intensity profiles behave over the different axial locations within the LLHC. All the four plots from the various models considered start from a higher value and fall as the axial location increases. This is pointing out that the swirl intensity decreases as the fluid nears the spigot of the LLHC. When the plots are fitted with some selected data from literature, Chang and Dhir's [7] model is seen to over predict the swirl intensity at $z / D_{c}<8$ but predict the data well at $z / D_{c}>15$. Gomez [9] and Mantilla [8] models fairly fit the data.

Fig. 3 is also made to show how the swirl intensity will behave at various designs by changing the characteristic diameter, Dc as 20, 30 and $45 \mathrm{~mm}$; like supposing we are having different LLHCs. From the figure, it is obvious that the larger the characteristic diameter the higher will be the swirl intensity. The reason of which may be due to the longer distance of swirl.

Investigations are made into the velocity profiles of the fluid within the LLHC. Fig. 4 shows the profile for the tangential velocity of the fluid droplets 
when each of the models is considered. The y-axis represents the cyclone axis whereas the $\mathrm{x}$-axis represents the radial position. All the models show the Rankine Vortex shape, the combination of forced vortex near the LLHC axis and the free vortex near the wall. When the figure was fitted with some field test data from DOWS operations, Gomez model [9] seems to give a better mathematical simulation of the tangential velocity compared to the others models. Using Gomez model, fig. 5 was made to show how droplet tangential velocity behaves at different axial locations. The results demonstrate that the highest tangential velocity component occurs at around $0.2 D_{c}$ in the selected axial locations $Z / D_{c}=5.5,10.5$ and 17.5.

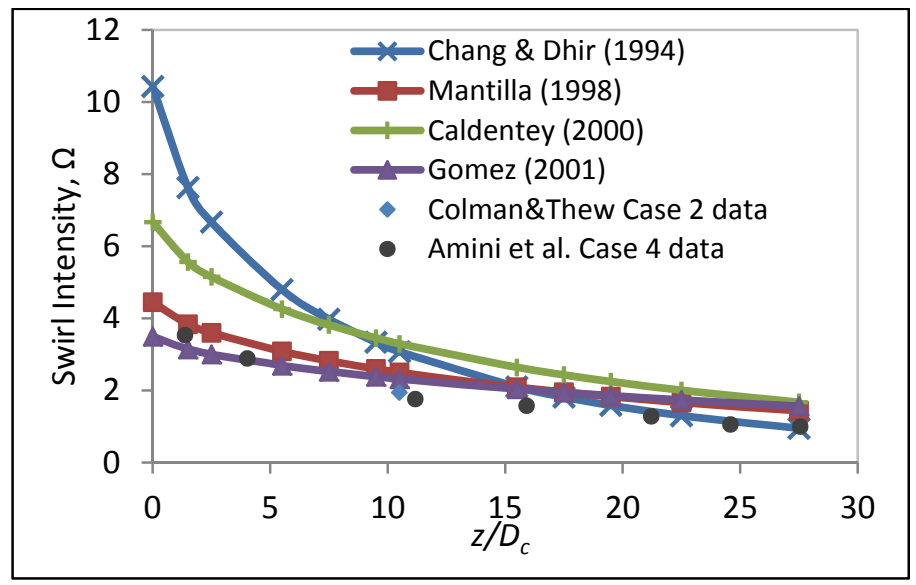

Figure 2: Swirl intensity comparison.

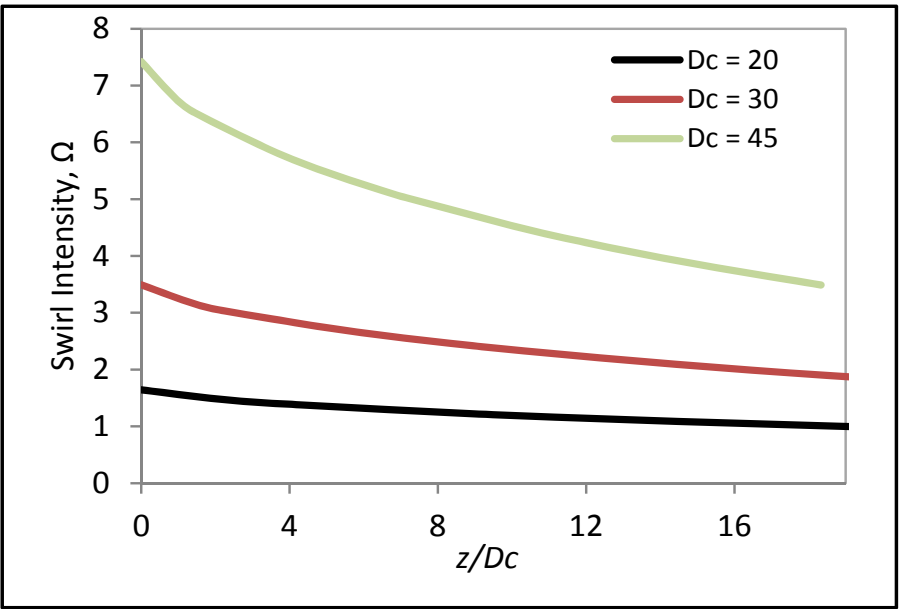

Figure 3: Swirl intensity at various characteristic diameters. 


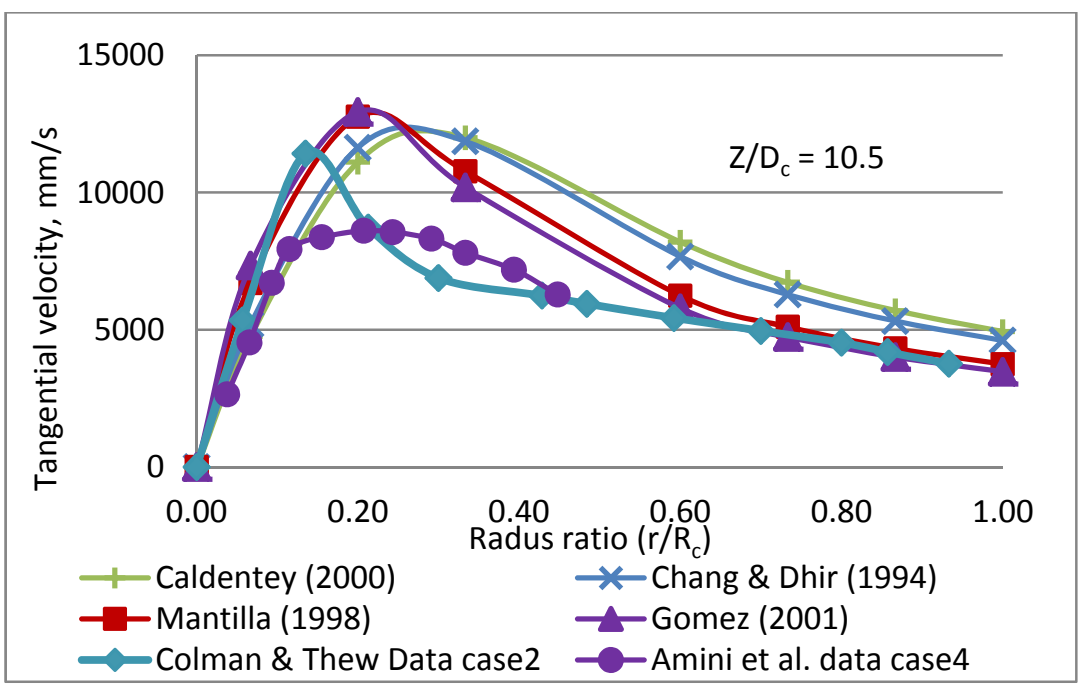

Figure 4: Tangential velocity profile as predicted from different models, at $z / D_{c}=10.5$.

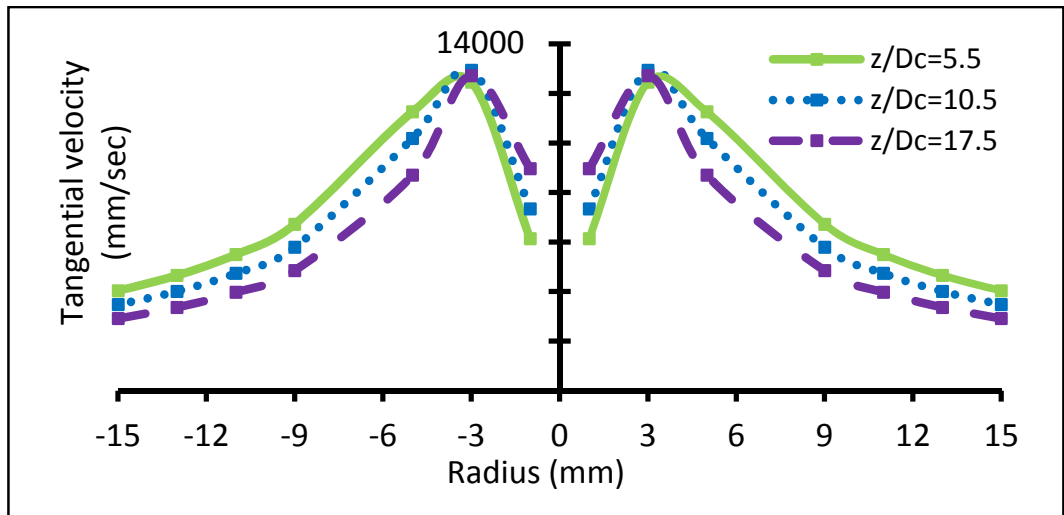

Figure 5: Tangential velocity profile at various axial locations.

The axial velocity profile for the flow within the LLHC is shown in fig. 6 . The positive values of the axial velocities correspond to downward flow while the negative values correspond to the reverse flow. The model matches well the data points for the downward flow and not good in the reverse flow region. However, in computing the separation efficiency of the LLHC, the reverse flow velocity is not so important but it is the flow reversal radius that matters.

Fig. 7 presents the combined effect of both tangential and axial velocities in the separation process. The tangential velocity is seen to be higher in magnitude than the axial velocity at various radii positions. This confirms the fact that the 
tangential velocity component is the most dominant component followed by the axial velocity component which is the second most dominant velocity component. At the reverse flow radius, the tangential velocity reaches almost its highest point with the axial velocity equal to zero.

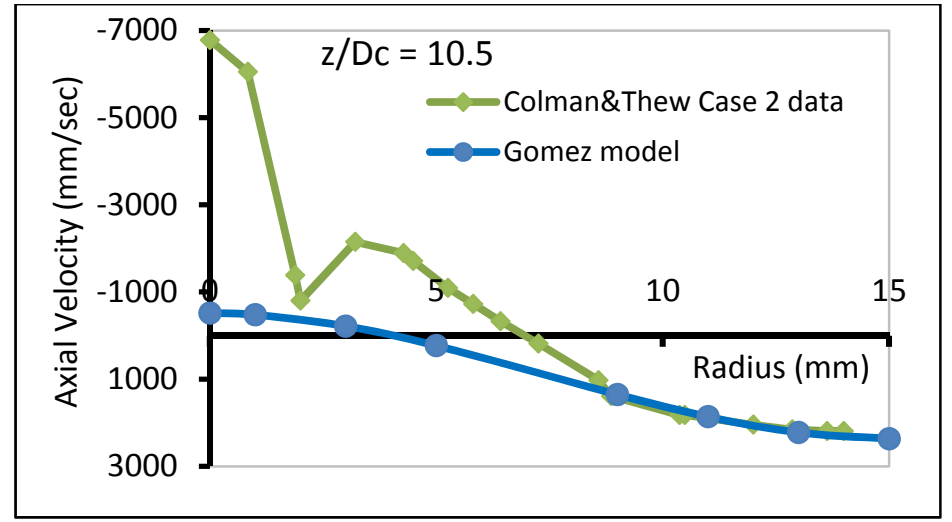

Figure 6: Axial velocity profile.

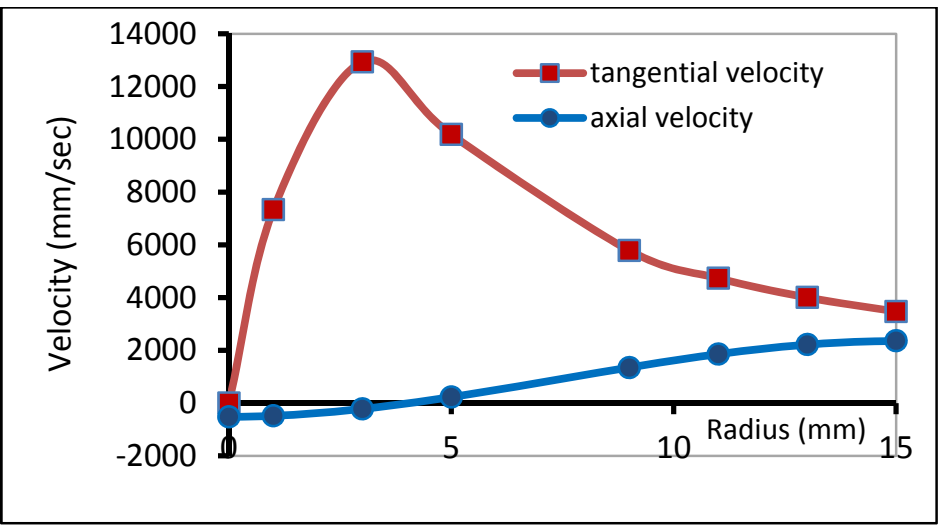

Figure 7: Effect of tangential and axial velocity.

\section{Conclusion}

This work has shown how relevant an in-depth knowledge about the hydrodynamics of the LLHC can be. It has given a clear view of what happens inside the LLHC. The swirl intensity increases with increasing characteristic diameter of the cyclone and always starts from a higher value and reduces in value with increasing axial location. The flow reversal radius is an important factor in the operation of the LLHC as it determines how the separation efficiency would be. Gomez model agrees with most of the experimental data 
obtained from literature. An experiment must therefore be performed using the proposed LLHC design modified after Colman and Thew and the data that will be obtained used to validate the mathematical correlations proposed by the aforementioned scholars.

Many wells that have been abandoned because of the production of higher water cuts can be reactivated by this technology.

\section{Acknowledgement}

The authors acknowledge Universiti Teknolgi PETRONAS for the financial and technical support to produce this paper under the research grant YUTP - FRG 015-3AA-A73.

\section{References}

[1] Chen, W., Zydek, N., and Parma, F., Evaluation of hydrocyclone models for practical applications, Chem. Eng. J., vol. 80, pp. 295-303, 2000.

[2] Bowers, B.E., Brownlee, R.F. and Schrenkel, P.J., Development of a downhole oil/water separation and reinjection system for offshore application, SPE Prod. \& Facilities, vol. 15, no. 2, pp. 115-122, 2000.

[3] Gomez, C., Caldentey, J., Wang, S., Gomez, L., Mohan, R. and Shoham, O., Oil/Water Separation in Liquid/Liquid Hydrocyclones (LLHC): Part 1 - Experimental Investigation, SPE J., pp. 353-361, 2002.

[4] Schubert, M.F., Advancements in Liquid Hydrocyclone Separation Systems, Offshore Technology Conf., pp. 497-506, 1992.

[5] Husveg, T., Johansen, O. and Bilstad, T., Operational Control of Hydrocyclones During Variable Produced Water Flow Rates - FrØy Case Study, SPE Prod. \& Operation, pp. 294-300, 2007.

[6] Caldentey, J., Gomez, C., Wang, S., Gomez, L., Mohan, R., and Shoham, O., Oil/Water Separation in Liquid/Liquid Hydrocyclones (LLHC): Part 2 - Mechanistic Modeling, SPE J., pp. 362-372, 2002.

[7] Chang, F. and Dhir, V.K., turbulent flow field in tangentially injected swirl flows in tubes, Int. J. Heat and Fluid flow, Vol. 15, No. 5, pp. 346356. 1994.

[8] Mantilla, I.S., Bubble Trajectory Analysis in Gas-Liquid Cylindrical Cyclone Separators, MSc dissertation, Dept. Petroleum Eng., University of Tulsa, 1998.

[9] Gomez, C.H., Oil-Water Separation in Liquid-Liquid Hydrocyclones (LLHC) Experiment and Modeling, MSc. dissertation, Dept. Petroleum Eng., University of Tulsa, 2001.

[10] Amini, D.M., Golkar, M., and Esmaeilzadeh, F., Mathematical modelling of a hydrocyclone for the down-hole oil-water separation (DOWS), Chem. Eng. Research and Design, vol. 90, pp. 2186-2195, 2012. 